\title{
Temperature heterogeneity correlates with intraspecific variation in physiological flexibility in a small endotherm
}

\author{
Maria Stager ${ }^{1 *}$, Nathan R. Senner ${ }^{2}$, David L. Swanson ${ }^{3}$, Matthew D. Carling ${ }^{4}$, Douglas K. Eddy ${ }^{4}$, \\ Timothy J. Grieves ${ }^{5}$, and Zachary A. Cheviron ${ }^{1}$
}

${ }^{1}$ Division of Biological Sciences, University of Montana, Missoula, MT, USA 59812

${ }^{2}$ Department of Biological Sciences, University of South Carolina, Columbia, SC USA 29208

${ }^{3}$ Department of Biology, University of South Dakota, Vermillion, SD USA 57069

${ }^{4}$ Department of Zoology and Physiology, University of Wyoming, Laramie, WY USA 82071

${ }^{5}$ Department of Biological Sciences, North Dakota State University, Fargo, ND USA 58102

*Corresponding author: mstager@mailbox.sc.edu

\begin{abstract}
Phenotypic flexibility allows individuals to reversibly modify trait values and theory predicts an individual's relative degree of flexibility positively correlates with the environmental heterogeneity it experiences. We tested this prediction by integrating surveys of population genetic and physiological variation with thermal acclimation experiments and indices of environmental heterogeneity in the Dark-eyed Junco (Junco byemalis) and its congeners. We combined measures of thermogenic capacity for $\sim 300$ individuals, $>21,000$ single nucleotide polymorphisms genotyped in 192 individuals, and laboratory acclimations replicated on five populations. We found that Junco populations: (1) differ in their thermal performance responses to temperature variation in situ; (2) exhibit intra-specific variation in their thermogenic flexibility in the laboratory that correlates with heterogeneity in their native thermal environment; and (3) harbor genetic variation that also correlates with temperature heterogeneity. These results provide comprehensive support that phenotypic flexibility corresponds with environmental heterogeneity and highlight its importance for coping with environmental change.
\end{abstract}

\section{INTRODUCTION}

Phenotypic plasticity - the ability of a single genotype to produce multiple trait values in response to an environmental cue - can be important for colonizing and persisting in novel environments $^{1-3}$. As a result, the role of plasticity in adaptation to environmental variation has received significant attention ${ }^{4-6}$. These studies have documented standing genetic variation in plastic responses ${ }^{7}$, and the potential for plasticity to evolve in response to natural selection ${ }^{8}$. Because adaptive plasticity should increase fitness in variable environments, theory predicts that the magnitude of plasticity that individuals exhibit should positively correlate with the amount of environmental heterogeneity they experience ${ }^{9-11}$.

Empirical evaluations of this prediction have provided conflicting results, but most have focused on traits that are developmentally plastic (i.e., those that undergo environmentally-induced but irreversible changes to a trait value during specific developmental windows). For instance, morphological plasticity varies with diet breadth among ecotypes of threespine stickleback ${ }^{12}$. Similarly, plasticity in development time positively correlates with spatial variation in pool-drying regimes in the common frog (Rana temporana) ${ }^{13}$. Analogous patterns have been shown in plants as well. In a bindweed (Convolvulus chilensis), plasticity in leaf morphology and functional traits varies with interannual variation in rainfall ${ }^{14}$. Conversely, multiple studies have found that plasticity for thermal tolerance limits is not associated with latitudinal or thermal seasonality in Drosophila ${ }^{15-17}$. The disparities among these studies may arise from differences in the relationship between the temporal scale over which an environment varies and the time required for phenotypic induction. If so, traits 
that can be modified repeatedly may be expected to respond to intra-annual variation more strongly than traits that are irreversible.

Unlike developmental plasticity, phenotypic flexibility — the ability to reversibly modify trait values - provides repeated opportunities to match phenotypes to environmental change across an individual's lifetime, especially in long-lived organisms ${ }^{18}$. Flexibility is predicted to evolve in environments characterized by frequent and predictable environmental variation ${ }^{19}$. Many morphological, physiological, and behavioral traits are flexible, and flexibility can represent an adaptive acclimatization response ${ }^{20}$. Determining the causes and consequences of variation in flexibility among individuals is therefore crucial to our understanding of adaptive evolution, and our ability to predict species' resilience to environmental change. Yet few empirical studies have tested whether geographic variation in the degree of flexibility is associated with spatial patterns of environmental heterogeneity (but see ref. ${ }^{21}$ ), and none have accounted for non-independence among populations (due to shared common ancestry and ongoing gene flow), which can obscure true relationships among variables of interest when analyzing intraspecific patterns of phenotypic variation $^{22}$.

If environmental heterogeneity influences variation in adaptive plasticity/flexibility across a species' range, we would therefore also expect to find that environmental heterogeneity structures population genetic variation as well. Patterns of environmentally segregating genetic variation can arise when local selective regimes influence the rates of gene flow among environmentally distinct habitats $^{23,24}$ and are not uncommon among widespread species ${ }^{25,26}$. No study, however, has tested whether the same environmental driver correlates with both genetic variation and variation in the degree of flexibility in a trait across a species' entire range.

To uncover the environmental drivers of geographic variation in phenotypic flexibility, we investigated the flexible capacity of a key physiological trait in a temperate songbird, the Dark-eyed Junco (Junco hyemalis). Juncos are particularly well suited to investigations of phenotypic flexibility due to the extensive phenotypic variation they exhibit and the broad range of environments they occupy $^{27}$. The $J$. byemalis lineage is comprised of five distinct morphotypes and 14 subspecies that inhabit a variety of habitats (Fig. S1) and exhibit conspicuous differences in life history, migratory tendency, physiology, size, song, plumage, and behavior ${ }^{27-29}$. This diversity is thought to have arisen since the most recent glacial maxima when the J. byemalis ancestor diverged from J. phaeonotus fulvescens of southern Mexico and subsequently expanded its range across North America ${ }^{30,31}$. While environmental factors have been shown to partition genetic variation within a subset of $J$. byemalis $\operatorname{taxa}^{32}$, the major J. hyemalis morphotypes do not exhibit strong population genetic differentiation ${ }^{31}$ suggesting that considerable phenotypic diversity persists in the face of high rates of gene flow. The role that environmental conditions play in driving the diversification of this lineage thus remain unclear.

Juncos have also been the subject of intense physiological study. Many J. hyemalis groups winter at temperate latitudes, and temperate environments place a premium on endogenous heat production in small homeothermic endotherms to maintain a relatively constant body temperature ${ }^{33}$. As a result, lab and field studies show that juncos increase their thermogenic capacity (the ability to generate heat; quantified as the peak metabolic rate under cold exposure, $\mathrm{M}_{\text {sum }}$ ) in the cold ${ }^{34-36}$. This heightened thermogenic capacity is associated with a reduced risk of hypothermia for juncos ${ }^{36,37}$, with a failure to achieve adequate thermogenic output having dire consequences for organismal fitness in small endotherms ${ }^{38-40}$. Increases in thermogenic performance are also accompanied by changes occurring at lower hierarchical levels of biological organization ${ }^{35,41-43}$. Thus, a higher thermogenic capacity may be energetically costly to maintain due to the additional metabolic machinery required to achieve elevated thermogenic performance ${ }^{44}$. Phenotypic flexibility in thermogenic capacity could therefore help mediate a balance between thermoregulation and its 
associated maintenance costs in response to fluctuating selective pressures ${ }^{45}$. Nonetheless, it is unknown whether an individual's capacity for thermogenic flexibility is influenced by the degree of thermal variability it experiences throughout the year.

We drew upon natural variation that exists across the Junco distribution to understand geographic variation in flexibility of thermogenic performance, and to test for associations between environmental heterogeneity, phenotypic flexibility, and population genetic structure. To do this, we first surveyed in situ geographic variation in Junco thermogenic capacity to determine which environmental indices structure variation in this trait. We then characterized fine-scale, range-wide population genetic structure within the Junco genus to determine whether it is influenced by the same climatic indices. Finally, we performed a laboratory acclimation experiment on five Junco populations that differ in their annual thermal regimes to test whether environmental heterogeneity predicts the degree of thermogenic flexibility. This approach allowed us to perform the first study that combines measures of physiological flexibility with indices of climatic variation while controlling for nonindependence among populations. We predicted that junco populations that experience greater seasonal temperature variation would exhibit higher thermogenic flexibility than those from more thermally stable regions. By combining these approaches, our results shed light on the ecological conditions that promote the evolution of increased flexibility and address long-standing hypotheses in the field of evolutionary biology.

\section{RESULTS AND DISCUSSION}

\section{Geographic variation in thermogenic performance}

Although several studies have characterized broad-scale interspecific patterns in endothermic thermogenic performance ${ }^{46-48}$, far less is known about the potential for or the underlying environmental correlates of intraspecific variation in thermogenic performance (but see refs. ${ }^{49-51}$ ). We assayed $\mathrm{M}_{\text {sum }}$ for 292 juncos at 8 sites across the U.S. and correlated recent weather conditions with patterns of in situ variation (Fig. 1). The number of individuals, number of sampling days, seasons, and years varied across sites with 86 total site-days of environmental variation and 5 morphotypes included in our dataset (Table S1).

Geographic variation in $\mathrm{M}_{\text {sum }}$ corresponded to environmental variation (Table S2). The most well-supported model included body mass $\left(\mathrm{M}_{\mathrm{b}}\right)$, daily temperature range $\left(\mathrm{T}_{\mathrm{d}_{-} \text {range }}\right)$, morphotype, and a $\mathrm{T}_{\mathrm{d} \_ \text {range }} \times$ morphotype interaction and explained $50 \%$ of the variation in $\mathrm{M}_{\text {sum }}$ (AICc $=883.4, w_{i}=$ 0.998). While $\mathrm{M}_{\mathrm{b}}$ positively correlated with $\mathrm{M}_{\text {sum }}(\beta=1.39 \pm 0.17, p<0.001)$, the model also showed a persistent effect of morphotype on $\mathrm{M}_{\text {sum }}$ after controlling for differences in $\mathrm{M}_{\mathrm{b}}$, with Oregon Juncos exhibiting the lowest and Slate-colored Juncos the highest $\mathrm{M}_{\text {sum }}$ values (Table S3). This could suggest local adaptation in thermogenic performance among populations. However, juncos that experienced larger $\mathrm{T}_{\mathrm{d}_{-} \text {range }}$ in the $7-8 \mathrm{~d}$ prior to capture also had the highest $\mathrm{M}_{\text {sum }}$, indicating that they may be responding to short-term heterogeneity in their thermal environment (Table S3). This is consistent with recent laboratory findings showing that $J$. h. montanus can make substantial changes to $\mathrm{M}_{\text {sum }}$ within one week of exposure to low temperature ${ }^{36}$. Moreover, we also found that the inclusion of the $T_{d_{-} \text {range }} \times$ morph interaction term substantially improved model fit indicating that some populations respond differentially to temperature variation in the wild (Fig. 2). Taken together, this suggests that Junco populations may differ in their physiological flexibility and that variation in the temperature range across their distribution may play an important role in shaping this flexibility.

\section{Genotype-environment associations}

The structure of the environment can also influence rates of gene flow among habitats and is therefore an important component determining the selective regime acting on local populations ${ }^{23,24}$. 
To understand how environmental variables might correspond to Junco population genetic variation, we genotyped 21,971 biallelic SNPs from 181 individuals that were selected to maximize geographic and environmental variance while representing all recognized Junco species/subspecies (Fig. S1). We first used a principal components analysis (PCA) to assess levels of underlying genetic variation across the radiation and found that the first two PC axes explained $7.1 \%$ of the total genetic variance. Major clusters identified by the PCA corresponded to the 'Sky Island' lineages of central America (J. vulcani), southern Mexico (J.p. alticola and J.p. fulvescens), and southern Baja (J.p. bairdi), as well as the Guadalupe Junco (J. insularis), with all other morphotypes grouping together (Fig. 3). The fact that all J. byemalis and northern J. phaeonotus taxa comprised one overlapping genetic cluster likely reflects the rapid expansion of this lineage over the last $\sim 20,000$ years ${ }^{30,31}$.

In instances where populations are not clearly distinguishable and environmental gradients are continuous, genotype-environment association methods can aid in the detection of signatures of natural selection ${ }^{52}$. In particular, redundancy analysis (RDA) is a powerful multivariate tool for identifying even weak correlations between genetic and environmental data ${ }^{53}$. We thus performed an RDA to quantify the population genetic variance that partitions with climatic indices while controlling for background genetic structure. Eight RDA axes explained $6.2 \%$ of the total genetic variance across Junco in our unconditioned model and 5.6\% in our conditioned model controlling for background population structure. Permutation tests confirmed the significance of the model in both cases $(p=0.001)$. The first 4 RDA axes were significant in the unconditioned model $(p<0.05)$, whereas the first 3 RDA axes were significant in the conditioned model. A variance partition analysis showed that temperature annual range explained more genetic variation than any other climatic variable that we tested in both the unconditioned $(0.52 \%)$ and conditioned models $(0.60 \%$; Table 1$)$. Additionally, we detected 751 outlier SNPs exhibiting associations with the first 3 axes in the conditioned model. Of these, 128 and 48 outlier SNPs corresponded most strongly to mean diurnal temperature range and annual temperature range, respectively. This complements our above finding that in situ physiological variation is also influenced by temperature range. However, it is not known whether these sites are involved in conferring flexibility and additional studies are necessary to reveal the genomic architecture underlying thermogenic flexibility.

Our results also show that several taxa overlap in orthogonal space (Fig. 3). Recent work by Friis et al. ${ }^{32}$ instead found that Oregon Junco taxa exhibited distinct environmental partitioning across their distribution. One reason for this difference may be that we included more Junco taxa in this study. In addition, Friis and colleagues sampled each taxon from a single site, which tends to confound environmental and geographic distance ${ }^{54}$. Our dataset encompasses far more environmental variation across varying geographic distances, and thus uniquely highlights the role of seasonal and diurnal climatic variation in structuring population genetic variation within and among Junco taxa.

\section{Flexible responses to temperature acclimation treatments}

To formally test whether phenotypic flexibility in thermogenic capacity correlated with environmental heterogeneity, we performed an acclimation experiment using individuals from five populations across the western U.S. Our ability to connect Junco populations to native climatic regimes is restricted by our limited knowledge of junco movements across the year, and we therefore focused on populations that likely remain resident to one narrow geographic area in order to reliably reconstruct their climatic histories. Based on the results of our in situ sampling, we selected focal populations to maximize variation in the annual temperature range $\left(T_{\text {range }}\right)$ they experienced. Genetic differentiation among populations $\left(\mathrm{F}_{\mathrm{ST}}\right)$ ranged from 0.019 to 0.051 (Table $\mathrm{S} 4$ ), while pairwise environmental and genetic distances did not covary (partial Mantel test: $r=-0.40, p=0.82$ ) allowing us to simultaneously tease apart the effects of both factors. 
Prior to acclimation, temperature treatment groups did not differ in $\mathrm{M}_{\text {sum }}(t=-1.10$, $\mathrm{df}=89$, $p=0.27)$ or $\mathrm{M}_{\mathrm{b}}(t=0.04, \mathrm{df}=89, p=0.97)$. However, both traits did positively correlate with native temperature range $\left(\mathrm{M}_{\text {sum: }} \beta=0.07 \pm 0.01, p<0.001 ; \mathrm{M}_{\mathrm{b}}: \beta=0.25 \pm 0.02, p<0.001\right)$. We therefore controlled for individual differences in pre-acclimation $\mathrm{M}_{\text {sum }}$ and $\mathrm{M}_{\mathrm{b}}$ in our subsequent analysis. Over the course of the experiment we found that $\Delta \mathrm{M}_{\text {sum }}$ (measured as the difference between postand pre-acclimation measures) was higher in larger and cold-acclimated birds $\left(\mathrm{M}_{\mathrm{b}}\right.$ : posterior mean $=$ $0.53,95 \% \mathrm{CI}=[0.03,1.00], p=0.03$; Cold Treatment: posterior mean $=0.62,95 \% \mathrm{CI}=[0.35$, $0.88], p<0.001)$. We also found an interaction between native $\mathrm{T}_{\text {range }}$ and treatment, such that populations from more variable climates exhibited the greatest increase in $\mathrm{M}_{\text {sum }}$ in the cold (posterior mean $=0.60,95 \% \mathrm{CI}=[0.07,1.14], p=0.03)$, while populations from less variable climates exhibited little or no change in $\mathrm{M}_{\text {sum }}$ (Fig. 4). Our results therefore clearly support our prediction that juncos from more thermally variable environments exhibit a greater capacity for thermogenic flexibility.

\section{Conclusions}

Our multifaceted approach integrated measures of population genetic variation with wholeorganism measures of physiological performance in both the lab and field, as well as indices of environmental variation to uncover the environmental drivers of geographic variation in phenotypic flexibility. We provide evidence that temperature variation drives patterns of intra-specific variation in thermal performance and found that junco populations responded differentially to weather cues in situ. This pattern was also replicated in the laboratory where thermogenic flexibility in juncos correlates with the heterogeneity of their native thermal environment. Moreover, range-wide population genetic variation also correlates with ambient thermal variation, providing evidence that environmental heterogeneity may be an important selective force driving junco population divergence. Together, these results suggest that physiological flexibility may play a key role in local adaptation in this broadly distributed genus.

While this result supports theory ${ }^{9-11}$, it contrasts with previous empirical studies in ectotherms that found that plasticity/flexibility in thermal physiology did not correspond to environmental heterogeneity ${ }^{15-17,55}$. While the cause of this disparity is unclear, one aspect that has largely been overlooked in previous studies is the role of historical demographic processes in shaping adaptive plasticity/flexibility. Gene flow ${ }^{56}$, colonization history ${ }^{57}$, population size ${ }^{58}$, and the standing genetic variation of founding individuals ${ }^{59}$ are all important factors shaping adaptive outcomes $^{26}$. For example, although gene flow can constrain adaptive divergence ${ }^{56}$, high gene flow among selective regimes is predicted to favor increased plasticity/flexibility in order to aid offspring that experience a dissimilar environment from their parents ${ }^{3,10,60}$. Comprehensive studies that simultaneously incorporate both contemporary ecological conditions and population demographic processes are necessary to fully understand the role of environment heterogeneity in structuring plasticity/flexibility.

There are also several biological differences between ectotherms and endotherms that may contribute to disparities in evolutionary patterns of phenotypic plasticity/flexibility. In general, many ectotherms rely on behavioral thermoregulatory mechanisms and possess a number of avoidance strategies (e.g., diapause, hibernation, or migration) that may be used to buffer against environmental extremes $^{61}$. Thus, the amount of thermal heterogeneity that an individual or population experiences may not correspond to broad-scale climatic patterns across the year. Endotherms maintain a relatively constant body temperature in comparison, despite sometimes large temperature differentials with their ambient environment ${ }^{33}$. While many endotherms also exhibit hibernation and migratory behaviors, small songbirds that reside in temperate regions year-round, like juncos, are 
particularly exposed to thermal heterogeneity ${ }^{45}$. These differences may lead to divergent selection pressures on flexibility and thermal performance traits among taxonomic groups.

This study greatly expands our knowledge of endothermic responses to environmental variation and their capacity for thermal acclimatization. Understanding flexibility in organismal thermal tolerances is important for predicting population dynamics ${ }^{62}$, making habitat delineations ${ }^{63}$, and modeling disease transmission ${ }^{64}$, all of which are especially relevant in light of ongoing global climate change. Although many recent macrophysiological approaches characterizing potential organismal responses to climatic change employ a single metric of thermal tolerance, effectively treating tolerance as a trait that is canalized and invariant across a species' range (e.g., refs ${ }^{55,65,66}$ ), our results highlight the capacity for populations to vary geographically in their physiological response to environmental cues. When coupled with datasets like ours, biophysical models that incorporate intraspecific patterns in acclimatization will improve our ability to predict organismal responses to climate warming.

\section{METHODS}

\section{In situ data and analysis}

In situ sampling

We captured juncos by mist net or potter trap at sites in Arizona (breeding season), Colorado (breeding), Illinois (wintering), Montana (breeding), New Mexico (wintering), New York (breeding), South Dakota (wintering), and Wyoming (breeding), spanning $16^{\circ}$ in latitude and $37^{\circ}$ in longitude (Fig. 1; Table S1). We classified individuals into known morphotypes based on plumage (Gray-headed, Oregon, Pink-sided, Slate-colored, and Yellow-eyed). These morphs have distinct breeding distributions that often encompass the ranges of multiple subspecies ${ }^{27,28,67}$; however, the wintering ranges overlap for many morphs.

\section{In situ metabolic assays}

We assayed $\mathrm{M}_{\text {sum }}$ of captured birds using open-flow respirometry near the site of capture. All measurements were made within $48 \mathrm{~h}$ of capture to avoid the effects of captivity on metabolic rates, though most were completed within $24 \mathrm{~h}$. $\mathrm{M}_{\mathrm{b}}$ was quantified before each measurement began. $\mathrm{M}_{\text {sum }}$ trials were conducted during the birds' light phase. A single individual was placed in a metabolic chamber in a dark, temperature-controlled environment. We pumped dry, cooled heliox gas $\left(21 \% \mathrm{O}_{2}, 79 \% \mathrm{He}\right)$ through the animal's chamber at a constant flow rate. We subsampled the outflow current, dried it (Drierite), scrubbed it of $\mathrm{CO}_{2}$ using ascarite, and dried it again before quantifying the $\mathrm{O}_{2}$ concentration using a FoxBox (Sable Systems). Trials were conducted using static cold exposure $\left(-5^{\circ} \mathrm{C}\right)$ for $\mathrm{CO}, \mathrm{IL}, \mathrm{MT}, \mathrm{NM}, \mathrm{NY}$, and WY birds and sliding cold exposure (starting at $0^{\circ} \mathrm{C}$ ) for $\mathrm{AZ}$ and SD birds; however, both methods have been shown to produce similar estimates of $\mathrm{M}_{\text {sum }}{ }^{68}$. Trials lasted until $\mathrm{O}_{2}$ consumption plateaued or declined for several minutes. We also sampled a blank chamber before and after trials to account for potential fluctuations in baseline, ambient air.

We used custom $\mathrm{R}$ scripts to quantify $\mathrm{M}_{\text {sum }}$ as the highest instant $\mathrm{O}_{2}$ consumption averaged over a 5-min period (https://github.com/Mstager/batch_processing_Expedata_files). We discarded measures characterized by large drift in baseline $\mathrm{O}_{2}$ (owing to ambient temperature fluctuations affecting the Fox Box) or inconsistent flow rates resulting in a total sample size of $n=292$ individuals. Following measurements, birds were subject to different fates: either released, exposed to acclimation experiments ${ }^{36}$, or immediately euthanized and deposited in museums (Table S1). Metabolic data from SD have been previously published ${ }^{69}$. 
To account for an individual's recent acclimatization history, we retrieved weather data associated with each collection site (rounded to the nearest hundredth of a degree latitude/longitude) from the DayMet dataset using the $\mathrm{R}$ package daymetr ${ }^{70}$. This dataset is composed of daily weather parameter estimates derived using interpolation and extrapolation from meteorological observations for $1 \mathrm{~km} \times 1 \mathrm{~km}$ gridded surfaces across North America ${ }^{71}$. We downloaded daily estimates of minimum temperature $\left(T_{\min }\right)$, maximum temperature $\left(T_{\max }\right)$, precipitation (prcp), water vapor pressure (wvp), and daylength (dayl) for the $14 \mathrm{~d}$ prior to and including each individual's capture date. We additionally calculated daily temperature range $\left(\mathrm{T}_{\mathrm{d}_{\_} \text {range }}\right)$ as $T_{\max }-T_{\min }$. We selected a conservative potential acclimatization window because, given their migratory nature, we do not know how long juncos were present at a site before sampling occurred, preventing us from incorporating extensive windows (e.g., months). Prior work in J. byemalis suggested a window of 7-14 d would be appropriate ${ }^{36,72}$. We therefore calculated running averages for each weather variable across acclimatization windows varying from 7-14 d preceding capture. We also retrieved elevation (elev) for each site using the package googleway ${ }^{73}$.

\section{Analyses for in situ data}

All analyses were conducted in $\mathrm{R}$ version 4.0.2 $2^{74}$. To determine whether junco $\mathrm{M}_{\text {sum }}$ varied with environmental variation, we constructed seven linear models with $\mathrm{M}_{\mathrm{b}}$, morphotype (morph), and a single environmental variable (elev or running averages of $T_{\min }, T_{\max }, T_{d_{-} \text {range, }}$ prcp, dayl, or wvp) as main effects. We first standardized continuous predictor variables according to ref. ${ }^{75}$ using the package arm. We then used $\mathrm{AIC}_{\mathrm{c}}$ values to evaluate differences in model fits among environmental variables and with that of a null model (including only $\mathrm{M}_{\mathrm{b}}$ and morph as predictors) in order to identify a single 'best' environmental variable and acclimatization window explaining variation in $\mathrm{M}_{\text {sum. }}$. As an indicator of differences in flexibility, we additionally tested for an interaction between the best environmental variable and morph to determine if populations differed in their response to environmental cues. To quantify the effect sizes of the interaction term, we reran the model with each morph as the reference.

\section{Population genetic data}

\section{Sampling, sequencing, and SNP generation}

For phylogeographic reconstruction, we obtained muscle tissue samples $(n=192)$ from museum specimens collected across the breeding distribution of all Junco species and subspecies (Table S5). This included 2-30 individuals per taxonomic unit sampled from 94 geographic localities representing the majority of U.S. counties, Canadian provinces, and Mexican states for which tissue samples exist (Fig. S1). We then employed restriction-site-associated DNA (RAD)-sequencing, which offers a reduced representation of the genome that can be mined for thousands of singlenucleotide polymorphisms (SNPs) among individuals ${ }^{76,77}$.

We extracted whole genomic DNA from each sample using a Qiagen DNeasy Blood and Tissue extraction Kit and prepared RAD-libraries according to ref. ${ }^{78}$ (protocol ver. 2.3 downloaded from dryad: https://datadryad.org/stash/dataset/doi:10.5061/dryad.m2271pf1). Briefly, we digested whole genomic DNA with two restriction enzymes (EcoRI and $M s e 1)$, ligated adaptor sequences with unique barcodes (8-10 bp) for each individual, performed PCR amplification, and then performed automated size selection of 300-400 bp fragments (Sage Science Blue Pippen). We split paired geographic samples between the two libraries such that all taxa were represented in each library of 96, pooled individuals. Libraries were sequenced on separate flow-cell lanes of an Illumina HiSeq 4000 at UC Berkeley's V.C. Genomics Sequencing Lab, yielding over 300 million, 100-nt single-end reads per lane. 
We demultiplexed reads, retained reads with intact EcoRI cut sites, removed inline barcodes and adapters, and performed quality filtering (removed Phred score $<10$ ) using process_radtags in STACKS ver. $2.1^{79}$. This resulted in final reads of $\mu=92 \mathrm{bp}$ in length and a mean of 2.05 million reads per individual. We removed 3 individuals in each lane that failed to sequence $(<100,000$ reads/individual, comprising $5 \mathrm{~J}$. hyemalis and $1 \mathrm{~J}$. insularis). We used bwa mem ${ }^{80}$ to align reads to the J. h. carolinensis genome ${ }^{32}$, which we downloaded from NCBI (Accession GCA_003829775.1). An average of $91 \%$ of reads mapped and mapping success did not differ among Junco species.

We executed the STACKS pipeline to call SNPs and exported one SNP per locus for loci present in all four Junco species in vcf format (populations: - 4 --write-random-snp), resulting in 178,574 SNPs. We further filtered the dataset using vcftools (ver. 0.1.16, Danecek et al. 2011). We removed sites with mean depth of coverage across all individuals $<5(--$ min-meanDP) and $>50(--$ maxmeanDP), minimum minor allele count $<3$ (--mac), more than $50 \%$ missing data (--max-missing), and characterized by indels (--remove-indels). We then removed $5 \mathrm{~J}$. hyemalis individuals with $>60 \%$ missing data (--remove). Finally, we removed sites with $>5 \%$ missing data (--max-missing) and filtered sites that departed from Hardy-Weinburg equilibrium assessed by species $(\not<0.001)$, resulting in 21,971 biallelic SNPs across 181 individuals. We exported the dataset in plink.raw format for downstream analysis.

\section{Genotype-environment association analyses}

To determine if environmental variation corresponded to genetic variation across Junco, we employed the SNP dataset in a redundancy analysis (RDA). RDA is a multivariate ordination technique that has been used to identify multiple candidate loci and several environmental predictors simultaneously ${ }^{53,82}$. Because RDA requires no missing data, we first imputed data for $n=68,311$ missing sites $(1.7 \%$ of total sites) using the most common genotype for the species at each site. We downloaded 19 interpolated monthly climate variables corresponding to the geographic origin of each specimen from WorldClim ${ }^{83}$ at a resolution of 2.5' using the $\mathrm{R}$ package raster ver. 3.3-13 ${ }^{84}$. We centered and standardized climate variables according to ref. $^{75}$. We excluded highly correlated variables $(r \geq 0.70)$ resulting in 8 variables retained: mean diurnal temperature range (BIO2, a measure of monthly temperature variation), temperature annual range (BIO7), mean temperature of the wettest quarter (BIO8), mean temperature of the driest quarter (BIO9), mean temperature of the warmest quarter (BIO10), precipitation of the driest month (BIO14), precipitation of the warmest quarter (BIO18), and precipitation of the coldest quarter (BIO19). We used these 8 climatic variables as predictors in RDAs executed with the package vegan ver. $2.5-6^{85}$. We performed both a simple RDA with no conditional treatment and a partial RDA conditioned on background population structure. For the latter, we summarized population genetic structure using the first two principal components from a principal component analysis of the imputed SNP data with the R package ade4 ver $1.7-15^{86}$. In both RDAs, we tested for, but did not find, multicollinearity among predictor variables (variance inflation factor $<5$ for all variables). We assessed the significance of the RDA models and of the constrained axes using an ANOVA-like permutation technique in vegan at $p \leq$ 0.05 ( $n=999$ and $n=99$ permutations, respectively). We estimated the proportion of variance explained by each climatic variable using variance partitioning ${ }^{87}$. We then identified candidate SNPs for environmental adaptation as those outside of a 3 standard deviation cutoff from the mean loading and characterized each candidate SNP by the predictor variable with which it had the strongest correlation (per ref. ${ }^{53}$ ).

\section{Acclimation experiments}

Population sampling for acclimation experiments 
We combined information gained from a literature search, eBird sightings, and expert opinion (pers. comm. Tom Martin) to identify five focal populations for phenotypic sampling that (1) were likely to be non-migratory, (2) represented different morphological subspecies, and (3) maximized variation in annual temperature range within the United States. These populations include the White-winged Junco (J. h. aikeni) of the Black Hills, a coastal population of Oregon Junco (J. h. shufeldti), a highland population of Red-backed Junco (J.h. dorsalis), a sky island population of Yellow-eyed Junco (J.p. palliatus), and a well-studied, urban population of Oregon Junco $(J \text {. h. thurberi })^{88}$. However, it is possible that some of these populations exhibit seasonal, altitudinal migrations within their geographic area of residence, e.g., J. p. palliatus ${ }^{89}$.

We captured $\leq 25$ individuals from each focal population. Capture periods differed for each population in order to increase the likelihood that targeted individuals were resident year-round, as well as due to time and permitting constraints. For instance, one partially migratory population $(J \cdot h$. aikeni) with distinct morphological features was caught in the winter to increase the likelihood that the individuals used were non-migratory. The other four populations, which bred in areas where other, morphologically similar juncos over-winter, were captured in the breeding season when other subspecies were not present. Specifically, J. h. shufeldti $(n=20)$ were captured 14-15 July 2018 in Coos and Douglas Counties, OR; J.p. palliatus $(n=24)$ were captured 27 July 2018 in Cochise County, AZ; J. h. dorsalis ( $n=25)$ were captured 30-31 July 2018 in Coconino County, AZ; J. h. aikeni $(n=15)$ were captured 6-9 March 2019 in Lawrence County, SD; and J. h. thurberi $(n=20)$ were captured 22-26 July 2019 in San Diego County, CA. In spite of these differences, we do not think capture season likely influenced acclimation ability because: (1) we subjected all individuals to a long adjustment period in order to erase prior acclimatization history before the experiment began (see below); and (2) the addition of a season term in our best model explaining variation in $\mathrm{M}_{\text {sum }}$ in situ did not improve model fit, suggesting that juncos are responding to proximate cues rather than making seasonal adjustments $\left(\beta_{\text {season }}=-0.11, p=0.64, \Delta \mathrm{AIC}_{\mathrm{c}}=1.97\right)$.

\section{Acclimation treatments}

Within days of capture, we ground-transported all birds to facilities at the University of Montana where birds were housed individually under common conditions $\left(23^{\circ} \mathrm{C}\right.$ with $12 \mathrm{~h}$ dark: 12 h light) for $\geq 8$ weeks $(\mu=63 \mathrm{~d}$, range $=57-71 \mathrm{~d})$. We had previously determined that a period of 6 wk is sufficient to reduce variation in metabolic traits among individuals (Fig. S2). Following this adjustment period, we assayed $\mathrm{M}_{\text {sum }}$ (see below). We allowed birds $\sim 24 \mathrm{~h}$ to recover and then randomly assigned individuals from each population into treatment groups and exposed them to either cold $\left(3^{\circ} \mathrm{C}\right)$ or control $\left(23^{\circ} \mathrm{C}\right)$ temperatures. Treatments lasted $21 \mathrm{~d}$ in duration. Constant $12 \mathrm{~h}$ dark: $12 \mathrm{~h}$ light days were maintained for the duration of the experiment, and food and water were supplied ad libitum. The diet consisted of a 2:1 ratio by weight of white millet and black oil sunflower seed, supplemented with ground dog food, live mealworms, and water containing vitamin drops (Wild Harvest D13123 Multi Drops). These experimental conditions were chosen based on previous work in $J$. h. hyemalis exposed to the same temperatures, which revealed substantial increases in $\mathrm{M}_{\text {sum }}$ over the same duration ${ }^{35}$.

Brood patches and cloacal protuberances were not present after the adjustment period. At the end of treatments, we euthanized individuals using cervical dislocation. Gonads, identified during dissection, were regressed in all but one individual (a male $J$. h. dorsalis) post-acclimation.

Eight individuals died during the capture-transport and adjustment periods $(1 \mathrm{~J}$. h. dorsalis, 4 J. h. shufeldti, $1 \mathrm{~J}$. h. thurberi, and $2 J$. p. palliatus). Additionally, one J. h. thurberi individual exhibited lethargy upon introduction to the cold treatment, died within the first $24 \mathrm{~h}$ of cold acclimation, and was removed from analyses. This resulted in a total sample size of $n=95$ individuals (Table S6). 


\section{Metabolic assays for acclimation experiments}

We quantified $\mathrm{M}_{\text {sum }}$ in a temperature-controlled cabinet using open-flow respirometry both before and after acclimation treatments as described above. We measured $\mathrm{M}_{\mathrm{b}}$ immediately before each assay. $\mathrm{M}_{\text {sum }}$ trials were conducted at $-5^{\circ} \mathrm{C}$ for pre-acclimation measures and $-15^{\circ} \mathrm{C}$ for postacclimation measures. Although this experimental temperature difference could have elicited higher post-acclimation $\mathrm{M}_{\text {sum }}$, we did not find variation in $\mathrm{M}_{\text {sum }}$ between the two time points in Control birds, suggesting that the two procedures provided similar levels of cold challenge (paired $\mathrm{t}$-test: $p=$ 0.60). Because trials occurred at various times throughout the day, we tested for, but did not find, a linear effect of trial start time on $\mathrm{M}_{\text {sum }}$ either before or after acclimation $\left(p_{\text {pre }}=0.54, p_{\text {post }}=0.94\right)$.

\section{Climate data for acclimation populations}

We reconstructed the annual thermal regime experienced by a population using interpolated monthly climate data downloaded from the WorldClim dataset ${ }^{83}$ with package raster. Specifically, we extracted the temperature annual range variable (BIO7) at a resolution of 2.5' for the site of capture (hereafter $\mathrm{T}_{\text {range }}$ ). Focal populations varied in $\mathrm{T}_{\text {range }}$ by $21^{\circ} \mathrm{C}$ (Fig. 1).

\section{Pair-wise genetic distance of acclimated populations}

To quantify genetic differentiation among acclimated populations, we extracted whole genomic DNA from the muscle tissue of acclimated individuals and prepared RAD-libraries as above with an additional PCR step (per protocol v. 2.6) ${ }^{78}$. We pooled all 95 individuals into a single flow-cell lane of an Illumina HiSeq X for sequencing at Novogene Corporation Inc. yielding 1 billion, 150-nt paired-end reads. We demultiplexed reads, removed inline barcodes and adaptors, and performed quality filtering (removed Phred score $<10$ ) using process_radtags. This resulted in final reads of $\mu=147 \mathrm{bp}$ in length and a mean of 7 million reads per individual. We removed 6 individuals that sequenced poorly $(<200,000$ reads/individual, comprising $1 \mathrm{~J}$. h. dorsalis, $1 \mathrm{~J}$. $p$. palliatus, $1 \mathrm{~J}$. h. shufeldti, and $3 \mathrm{~J}$. h. thurberi). We aligned reads to the J. h. carolinensis genome with bwa mem (51\% of reads mapped). We then sorted, merged, and removed read duplicates with samtools ver. $1.3^{90}$. We used the STACKS pipeline to call SNPs from loci present in all 5 populations and exported one SNP per locus in vef format resulting in 282,929 SNPs (populations: - $p$--write-random$\operatorname{snp})$.

We filtered the dataset and estimated patterns of genetic differentiation using vcftools. We removed sites with mean depth of coverage across all individuals $<20$ (--min-meanDP) and $>300$ (-max-meanDP), minor allele frequency $<5 \%(--$ min-maf), and $>10 \%$ missing data (--max-missing), and removed indels (--remove-indels), resulting in 1069 biallelic SNPs across 89 individuals. Finally, we estimated pairwise $\mathrm{F}_{\mathrm{ST}}$ among the focal taxa by calculating weighted Weir's theta ${ }^{91}$.

\section{Analyses for acclimation data}

We first performed a partial mantel test to ascertain that environmental and genetic distances do not covary among our sampling sites. We calculated pairwise genetic distance as $\mathrm{F}_{\text {ST }} /\left(1-\mathrm{F}_{\text {ST }}\right)$. We estimated pairwise environmental differences among the five sites as the Euclidean distance for 5 WorldClim variables (after removing redundant variables at $r \geq 0.70$ from the original 19 WorldClim variables). We simultaneously controlled for geographic distance, estimated as pairwise geodesic distance among sampling sites with the package geosphere $e^{92}$. We then employed these matrices of pairwise genetic, environmental, and geographic distance in a partial mantel test with the package $\operatorname{vegan}^{85}$. 
We verified that phenotypic differences did not exist among treatment groups before acclimations began using t-tests for $\mathrm{M}_{\mathrm{b}}$ and $\mathrm{M}_{\text {sum. }}$. We also used linear regression to quantify the effects of temperature range on pre-acclimation trait values. To evaluate whether environmental variation corresponded with flexibility, we related climatic data to $\mathrm{M}_{\text {sum }}$ for each population while simultaneously incorporating population demography. We used Markov Chain Monte Carlo generalized linear mixed models that allow for Bayesian approaches ${ }^{22,93}$ with the $M C M C g l m m$ package $^{94}$. We constructed a model to explain variation in thermogenic flexibility $\left(\Delta \mathrm{M}_{\text {sum }}\right.$; postminus pre-acclimation $\mathrm{M}_{\text {sum }}$ ) with pre-acclimation $\mathrm{M}_{\mathrm{b}}$, temperature treatment, $\mathrm{T}_{\text {range, and treatment }} \times$ $\mathrm{T}_{\text {range }}$ interaction as main effects and pairwise $\mathrm{F}_{\text {ST }}$ as a random effect. We standardized all continuous predictor variables according to ${ }^{75}$. We used default priors and ran the model for 1,000,000 iterations with a burn-in of 10,000 and a thinning interval of 100. We examined the resulting trace plots to verify proper convergence.

\section{ETHICS}

This work was completed with approval from the U.S. Fish and Wildlife Service (MB84376B-1 to M.S.; MB01543B-0 to Z.A.C.; MB758442 to D.L.S.; MB06336A-4 to M.D.C.; MB45239B-0 to T.J.G.; MB757670-1 to David Winkler; and MB094297-0 to Christopher Witt,), the State of Arizona Game and Fish Department (E19253811 to M.S. and SP590760 and SP707897 to D.L.S.), the State of California Department of Fish and Wildlife (13971 to M.S.), Colorado Parks and Wildlife (10TRb2030A15 to M.D.C.), the Illinois Department of Natural Resources (NH13.5667 to Z.A.C.), the Montana Department of Fish Wildlife and Parks (2016-013 and 2017067-W to M.S.), the New Mexico Department of Game \& Fish (\#3217 to Christopher Witt), the New York State Division of Fish, Wildlife, \& Marine Resources (LCP 1477 to David Winkler), the Oregon Department of Fish and Wildlife (108-18 to M.S.), the State of South Dakota Department of Game, Fish, and Parks (19-13 to M.S. and 06-03, 07-02, 08-03 to D.L.S.), Wyoming Game and Fish (754 to M.D.C.), and the Institutional Animal Care and Use Committees at Cornell University (2001-0051 to David Winkler), the University of Illinois (13385 to Z.A.C.), the University of Montana (010-16ZCDBS-020916 and 030-18ZCDBS-052918 to Z.A.C.), the University of South Dakota (03-08-06-08B to D.L.S), and the University of Wyoming (A-3216-01 to M.D.C.).

\section{ACKNOWLEDGMENTS}

We are indebted to the many natural history museums that contributed tissue loans to this project, including John Klicka and the University of Washington Burke Museum, the American Museum of Natural History, the Cleveland Museum of Natural History, the Cornell University Museum of Vertebrates, the Field Museum of Natural History, the Louisiana State University Museum of Natural Science, the Midwest Museum of Natural History, the Museum of Southwest Biology, the Museum of Vertebrate Zoology, the New York State Museum, the Royal Alberta Museum, the San Diego Natural History Museum, the Smithsonian National Museum of Natural History, the University of Alaska Museum, the University of Montana Zoological Museum, and the University of Wyoming Museum of Vertebrates. We are very thankful to the many hands that provided help and logistical assistance collecting birds: Gregory Toreev, Cole Wolf, Trey Sasser, Phred Benham, Nick Sly, Henry Pollock, Phil Unitt, Chris Witt, Andy Johnson, Blair Wolf, Eric Gulson, David Winkler, Charles Dardia, Link Smith, Pamela Yeh, Eleanor Diamant, Kevin Burns, and Point Loma Nazarene University. We are grateful to the field stations that hosted our work: UNM Sevilleta Field Station, UW-NPS Research Station, Mt. Evans Field Station, and the Southwestern Research Station. We also thank Brett Addis, Rena Schweizer, and Thom Nelson for guidance with RAD processing and pop gen analysis, and the Cheviron lab for feedback on an earlier version of this manuscript. 


\section{FUNDING}

This work was supported by funds from the American Museum of Natural History Chapman Fund, the American Philosophical Society Lewis and Clark Fund, the Explorers Club, the Illinois Ornithological Society, the Nuttall-Ornithological Society, Sigma Xi, the Society for Integrative and Comparative Biology, the Society of Systematic Biologists, the University of Illinois Graduate School, the University of Illinois School of Integrative Biology, and the Wilson Ornithological Society (to M.S.); the University of Montana (startup to Z.A.C). M.S. was supported by the National Science Foundation Graduate Research Fellowship Program, P.E.O. International, and the University of Montana Graduate School Bertha Morton Fellowship.

\section{AUTHOR CONTRIBUTIONS}

M.S. and Z.A.C. conceived of the study; M.D.C., D.K.E., and N.R.S. helped perform field work; D.L.S. performed in situ measurements in AZ and SD; T.J.G. provided sampling permits; M.S. performed all other data collection and analyses and drafted the manuscript; all authors contributed edits to the manuscript.

\section{DATA AVAILABILITY}

Raw reads have been deposited in the NCBI Sequence Read Archive (PRJNA678344). Phenotypic data are included in the Supplementary Materials. All associated scripts are deposited on github (https://github.com/Mstager/junco_flexibility_scripts). 


\section{REFERENCES}

1. Price, T. D., Qvarnström, A. \& Irwin, D. E. The role of phenotypic plasticity in driving genetic evolution. Proc. R. Soc. Lond. B 270, 1433-1440 (2003).

2. Ghalambor, C. K., McKAY, J. K., Carroll, S. P. \& Reznick, D. N. Adaptive versus non-adaptive phenotypic plasticity and the potential for contemporary adaptation in new environments. Funct Ecology 21, 394-407 (2007).

3. Crispo, E. Modifying effects of phenotypic plasticity on interactions among natural selection, adaptation and gene flow. Journal of Evolutionary Biology 21, 1460-1469 (2008).

4. Tienderen, P. H. V. Evolution of Generalists and Specialist in Spatially Heterogeneous Environments. Evolution 45, 1317 (1991).

5. West-Eberhard, M. J. Developmental Plasticity and Evolution. (Oxford University Press, 2003).

6. Scheiner, S. M. The genetics of phenotypic plasticity. XII. Temporal and spatial heterogeneity. Ecol Evol 3, 4596-4609 (2013).

7. Pigliucci, M. Evolution of phenotypic plasticity: where are we going now? Trends in Ecology \& Evolution 20, 481-486 (2005).

8. Nussey, D. H., Postma, E., Gienapp, P. \& Visser, M. E. Selection on heritable phenotypic plasticity in a wild bird population. Science 310, 304-306 (2005).

9. Moran, N. A. The evolutionary maintenance of alternative phenotypes. The American Naturalist 139, 971-989 (1992).

10. Sultan, S. E. \& Spencer, H. G. Metapopulation structure favors plasticity over local adaptation. The American Naturalist 160, 271-283 (2002).

11. Ernande, B. \& Dieckmann, U. The evolution of phenotypic plasticity in spatially structured environments: implications of intraspecific competition, plasticity costs and environmental characteristics. Journal of Evolutionary Biology 17, 613-628 (2004).

12. Day, T., Pritchard, J. \& Schluter, D. A Comparison of Two Sticklebacks. Evolution 48, 17231734 (1994).

13. Lind, M. I. \& Johansson, F. The degree of adaptive phenotypic plasticity is correlated with the spatial environmental heterogeneity experienced by island populations of Rana temporaria. Journal of Evolutionary Biology 20, 1288-1297 (2007).

14. Gianoli, E. \& González-Teuber, M. Environmental heterogeneity and population differentiation in plasticity to drought in Convolvulus chilensis (Convolvulaceae). Evol Ecol 19, 603-613 (2005).

15. Overgaard, J., Kristensen, T. N., Mitchell, K. A. \& Hoffmann, A. A. Thermal tolerance in widespread and tropical drosophila species: does phenotypic plasticity increase with latitude? The American Naturalist 178, S80-S96 (2011).

16. van Heerwaarden, B., Lee, R. F. H., Overgaard, J. \& Sgrò, C. M. No patterns in thermal plasticity along a latitudinal gradient in Drosophila simulans from eastern Australia. Journal of Evolutionary Biology 27, 2541-2553 (2014).

17. Sørensen, J. G., Kristensen, T. N. \& Overgaard, J. Evolutionary and ecological patterns of thermal acclimation capacity in Drosophila: is it important for keeping up with climate change? Current Opinion in Insect Science 17, 98-104 (2016).

18. Piersma, T. \& Drent, J. Phenotypic flexibility and the evolution of organismal design. Trends in Ecology \& Evolution 18, 228-233 (2003).

19. Botero, C. A., Weissing, F. J., Wright, J. \& Rubenstein, D. R. Evolutionary tipping points in the capacity to adapt to environmental change. Proc Natl Acad Sci USA 112, 184-189 (2015).

20. Piersma, T. \& van Gils, J. A. The Flexible Phenotype: A Body-centred Integration of Ecology, Physiology, and Behaviour. (Oxford University Press, 2011). 
21. Cavieres, G. \& Sabat, P. Geographic variation in the response to thermal acclimation in rufouscollared sparrows: are physiological flexibility and environmental heterogeneity correlated? Functional Ecology 22, 509-515 (2008).

22. Stone, G. N., Nee, S. \& Felsenstein, J. Controlling for non-independence in comparative analysis of patterns across populations within species. Phil. Trans. R. Soc. B 366, 1410-1424 (2011).

23. Lenormand, T. Gene flow and the limits to natural selection. Trends in Ecology \& Evolution 17, 183-189 (2002).

24. Kawecki, T. J. \& Ebert, D. Conceptual issues in local adaptation. Ecology Letters 7, 1225-1241 (2004).

25. Schweizer, R. M. et al. Targeted capture and resequencing of 1040 genes reveal environmentally driven functional variation in grey wolves. Molecular Ecology 25, 357-379 (2016).

26. Benham, P. M. \& Cheviron, Z. A. Population history and the selective landscape shape patterns of osmoregulatory trait divergence in tidal marsh Savannah sparrows (Passerculus sandwichensis). Evolution 74, 57-72 (2020).

27. Nolan, Jr., V. et al. Dark-eyed Junco (Junco hyemalis). Birds N. Am. (2002) doi:10.2173/bna.716.

28. Miller, A. H. Speciation in the avian genus Junco. vol. 44 (University of California Press, 1941).

29. Ketterson, E. D. \& Atwell, J. W. Snowbird: integrative biology and evolutionary Ddversity in the Junco. (University of Chicago Press, 2016).

30. Milá, B., McCormack, J. E., Castañeda, G., Wayne, R. K. \& Smith, T. B. Recent postglacial range expansion drives the rapid diversification of a songbird lineage in the genus Junco. Proc. R. Soc. B 274, 2653-2660 (2007).

31. Friis, G., Aleixandre, P., Rodríguez-Estrella, R., Navarro-Sigüenza, A. G. \& Milá, B. Rapid postglacial diversification and long-term stasis within the songbird genus Junco: phylogeographic and phylogenomic evidence. Mol Ecol 25, 6175-6195 (2016).

32. Friis, G. et al. Genome-wide signals of drift and local adaptation during rapid lineage divergence in a songbird. Mol Ecol 27, 5137-5153 (2018).

33. McNab, B. The physiological ecology of vertebrates: A View from Energetics. in Copeia vol. 2002 (2002).

34. Swanson, D. L. Seasonal variation in cold hardiness and peak rates of cold-induced thermogenesis in the Dark-Eyed Junco (Junco hyemalis). The Auk 107, 6 (1990).

35. Swanson, D., Zhang, Y., Liu, J.-S., Merkord, C. L. \& King, M. O. Relative roles of temperature and photoperiod as drivers of metabolic flexibility in dark-eyed juncos. Journal of Experimental Biology 217, 866-875 (2014).

36. Stager, M., Senner, N. R., Tobalske, B. W. \& Cheviron, Z. A. Body temperature maintenance acclimates in a winter-tenacious songbird. J Exp Biol 223, jeb221853 (2020).

37. Swanson, D. Are summit metabolism and thermogenic endurance correlated in winteracclimatized passerine birds? Journal of Comparative Physiology B: Biochemical, Systemic, and Environmental Physiology 171, 475-481 (2001).

38. Hayes, J. P. \& O'Connor, C. S. Natural selection on thermogenic capacity of high-altitude deer mice. Evolution 53, 1280-1287 (1999).

39. Petit, M., Clavijo-Baquet, S. \& Vézina, F. Increasing winter maximal metabolic rate improves intrawinter survival in small birds. Physiological and Biochemical Zoology 90, 166-177 (2017).

40. Latimer, C. E., Cooper, S. J., Karasov, W. H. \& Zuckerberg, B. Does habitat fragmentation promote climate-resilient phenotypes? Oikos 127, 1069-1080 (2018).

41. Swanson, D. L. Seasonal variation of vascular oxygen transport in the Dark-Eyed Junco. The Condor 92, 62-66 (1990). 
42. Stager, M., Swanson, D. L. \& Cheviron, Z. A. Regulatory mechanisms of metabolic flexibility in the dark-eyed junco (Junco hyemalis). Journal of Experimental Biology 218, 767-777 (2015).

43. Stager, M. \& Cheviron, Z. A. Is there a role for sarcolipin in avian facultative thermogenesis in extreme cold? Biology Letters 16, 20200078 (2020).

44. Vezina, F. et al. Consequences of being phenotypically mismatched with the environment: rapid muscle ultrastructural changes in cold-shocked black-capped chickadees (Poecile atricapillus). American Journal of Physiology-Regulatory, Integrative and Comparative Physiology 318, R274-R283 (2020).

45. Swanson, D. L. Seasonal metabolic variation in birds: functional and mechanistic correlates. in Current Ornithology Volume 17 (ed. Thompson, C. F.) 75-129 (Springer New York, 2010). doi:10.1007/978-1-4419-6421-2_3.

46. Naya, D. E., Spangenberg, L., Naya, H. \& Bozinovic, F. Latitudinal Patterns in Rodent Metabolic Flexibility. The American Naturalist 179, E172-E179 (2012).

47. Stager, M. et al. Disentangling environmental drivers of metabolic flexibility in birds: the importance of temperature extremes versus temperature variability. Ecography 39, 787-795 (2016).

48. Buckley, L. B., Khaliq, I., Swanson, D. L. \& Hof, C. Does metabolism constrain bird and mammal ranges and predict shifts in response to climate change? Ecol Evol ece3.4537 (2018) doi:10.1002/ece3.4537.

49. Dawson, W. R., Marsh, R. L., Buttemer, W. A. \& Carey, C. Seasonal and geographic variation of cold resistance in House Finches Carpodacus mexicanus. Physiological Zoology 56, 353-369 (1983).

50. O'Connor, T. P. Geographic Variation in Metabolic Seasonal Acclimatization in House Finches. The Condor 98, 371-381 (1996).

51. Olson, J. R., Cooper, S. J., Swanson, D. L., Braun, M. J. \& Williams, J. B. The relationship of metabolic performance and distribution in Black-Capped and Carolina Chickadees. Physiological and Biochemical Zoology 83, 263-275 (2010).

52. Jones, M. R. et al. Integrating landscape genomics and spatially explicit approaches to detect loci under selection in clinal populations. Evolution 67, 3455-3468 (2013).

53. Forester, B. R., Lasky, J. R., Wagner, H. H. \& Urban, D. L. Comparing methods for detecting multilocus adaptation with multivariate genotype-environment associations. Molecular Ecology 27, 2215-2233 (2018).

54. Wang, I. J. \& Bradburd, G. S. Isolation by environment. Molecular Ecology 23, 5649-5662 (2014).

55. Gunderson, A. R. \& Stillman, J. H. Plasticity in thermal tolerance has limited potential to buffer ectotherms from global warming. Proc. R. Soc. B 282, 20150401 (2015).

56. Riechert, S. E. Investigation of potential gene flow limitation of behavioral adaptation in an aridlands spider. Behav Ecol Sociobiol 32, 355-363 (1993).

57. Beall, C. M. Two routes to functional adaptation: Tibetan and Andean high-altitude natives. Proceedings of the National Academy of Sciences 104, 8655-8660 (2007).

58. Leimu, R. \& Fischer, M. A meta-analysis of local adaptation in plants. PLoS ONE 3, e4010 (2008).

59. Barrett, R. \& Schluter, D. Adaptation from standing genetic variation. Trends in Ecology \& Evolution 23, 38-44 (2008).

60. Lind, M. I., Ingvarsson, P. K., Johansson, H., Hall, D. \& Johansson, F. Gene flow and selection on phenotypic plasticity in an island system of Rana temporaria. Evolution 65, 684-697 (2011).

61. Kearney, M., Shine, R. \& Porter, W. P. The potential for behavioral thermoregulation to buffer "cold-blooded" animals against climate warming. Proc Natl Acad Sci U S A 106, 3835-3840 (2009). 
62. McFarlane, S. E., Ålund, M., Sirkiä, P. M. \& Qvarnström, A. Difference in plasticity of resting metabolic rate - the proximate explanation to different niche breadth in sympatric Ficedula flycatchers. Ecology and Evolution 8, 4575-4586 (2018).

63. Du, W. G. D. G., Shou, L. S. \& Shen, J. Y. S. Y. Habitat selection in two sympatric Chinese skinks, Eumeces elegans and Sphenomorphus indicus: do thermal preferences matter? Canadian Journal of Zoology (2006) doi:10.1139/z06-116.

64. Miazgowicz, K. L. et al. Age influences the thermal suitability of Plasmodium falciparum transmission in the Asian malaria vector Anopheles stephensi. Proceedings of the Royal Society B: Biological Sciences 287, 20201093 (2020).

65. Sunday, J. M. et al. Thermal-safety margins and the necessity of thermoregulatory behavior across latitude and elevation. Proceedings of the National Academy of Sciences 111, 5610-5615 (2014).

66. Riddell, E. A., Iknayan, K. J., Wolf, B. O., Sinervo, B. \& Beissinger, S. R. Cooling requirements fueled the collapse of a desert bird community from climate change. Proc Natl Acad Sci USA 116, 21609-21615 (2019).

67. Sullivan, K. A. Yellow-eyed Junco - Junco phaeonotus - v1.1 from Birds of North America Birds of the World. https://birdsoftheworld.org/bow/historic/bna/yeejun/1.1/introduction (2018).

68. Swanson, D. L., Drymalski, M. W. \& Brown, J. R. Sliding vs static cold exposure and the measurement of summit metabolism in birds. Journal of Thermal Biology 21, 221-226 (1996).

69. Swanson, D. L., Thomas, N. E., Liknes, E. T. \& Cooper, S. J. Intraspecific correlations of basal and maximal metabolic rates in birds and the aerobic capacity model for the evolution of endothermy. PLoS ONE 7, e34271 (2012).

70. Hufkens, K., Basler, D., Milliman, T., Melaas, E. \& Richardson, A. An integrated phenology modelling framework in R. Methods in Ecology and Evolution 9, 1276-1285 (2018).

71. Thornton, P. E. et al. Daymet: Daily Surface Weather Data on a 1-km Grid for North America, Version 3. ORNL DAAC (2016) doi:https://doi.org/10.3334/ORNLDAAC/1328.

72. Swanson, D. L. \& Olmstead, K. L. Evidence for a proximate influence of winter temperature on metabolism in passerine biirds. Physiological and Biochemical Zoology 72, 566-575 (1999).

73. Cooley, D., decode_pl), P. B. (Author of c++ \& pacakge), R. (Functions written for the L. googleway: Accesses Google Maps APIs to Retrieve Data and Plot Maps. (2018).

74. R Core Team. R: The R project for statistical computing. R Foundation for Statistical Computing, Vienna, Austria https://www.r-project.org/ (2018).

75. Gelman, A. Scaling regression inputs by dividing by two standard deviations. Statist. Med. 27, 2865-2873 (2008).

76. Davey, J. W. \& Blaxter, M. L. RADSeq: next-generation population genetics. Briefings in Functional Genomics 9, 416-423 (2010).

77. Baird, N. A. et al. Rapid SNP discovery and genetic mapping using sequenced RAD markers. PLOS ONE 3, e3376 (2008).

78. Parchman, T. L. et al. Genome-wide association genetics of an adaptive trait in lodgepole pine: association mapping of serotiny. Molecular Ecology 21, 2991-3005 (2012).

79. Catchen, J. M., Amores, A., Hohenlohe, P., Cresko, W. \& Postlethwait, J. H. Stacks : Building and genotyping loci De Novo from short-read sequences. G3 1, 171-182 (2011).

80. Li, H. Aligning sequence reads, clone sequences and assembly contigs with BWA-MEM. ArXiv 1303, (2013).

81. Danecek, P. et al. The variant call format and VCFtools. Bioinformatics 27, 2156-2158 (2011).

82. Forester, B. R., Jones, M. R., Joost, S., Landguth, E. L. \& Lasky, J. R. Detecting spatial genetic signatures of local adaptation in heterogeneous landscapes. Molecular Ecology 25, 104-120 (2016). 
83. Hijmans, R. J., Cameron, S. E., Parra, J. L., Jones, P. G. \& Jarvis, A. Very high resolution interpolated climate surfaces for global land areas. nternational Journal of Climatology 25, 1965-1978 (2005).

84. Hijmans, R. J. et al. raster: Geographic Data Analysis and Modeling. (2020).

85. Oksanen, J. et al. vegan: Community Ecology Package. (2019).

86. Dray, S. \& Dufour, A.-B. The ade4 Package: Implementing the duality diagram for ecologists. Journal of Statistical Software 22, 1-20 (2007).

87. Borcard, D., Legendre, P. \& Drapeau, P. Partialling out the spatial component of ecological variation. Ecology 73, 1045-1055 (1992).

88. Yeh, P. J. \& Price, T. D. Adaptive phenotypic plasticity and the successful colonization of a novel environment. The American Naturalist 164, 531-542 (2004).

89. Lundblad, C. G. \& Conway, C. J. Variation in selective regimes drives intraspecific variation in life-history traits and migratory behaviour along an elevational gradient. Journal of Animal Ecology 89, 397-411 (2020).

90. Li, H. et al. The Sequence Alignment/Map format and SAMtools. Bioinformatics 25, 2078-2079 (2009).

91. Weir, B. S. \& Cockerham, C. C. Estimating f-statistics for the analysis of population structure. Evolution 38, 1358-1370 (1984).

92. Hijmans, R. J., Williams, E. \& Vennes, C. geosphere: Spherical Trigonometry. (2019).

93. Hadfield, J. D. \& Nakagawa, S. General quantitative genetic methods for comparative biology: phylogenies, taxonomies and multi-trait models for continuous and categorical characters. Journal of Evolutionary Biology 23, 494-508 (2010).

94. Hadfield, J. D. MCMC Methods for multi-response generalized linear mixed models: The MCMCglmm R Package. Journal of Statistical Software 33, 1-22 (2010). 
Table 1. Genetic variance across 21,971 SNPs partitioned by 8 climatic variables in an unconditioned RDA and an RDA conditioned on background structure.

\begin{tabular}{lcc}
\multicolumn{1}{c}{ Variable } & $\begin{array}{c}\text { Unconditioned } \\
\text { Adj. } \mathbf{R}^{\mathbf{2}}\end{array}$ & $\begin{array}{c}\text { Conditioned } \\
\mathbf{A d j} . \mathbf{R}^{\mathbf{2}}\end{array}$ \\
\hline \hline Diurnal Temperature Range & 0.0026 & 0.0034 \\
Temperature Annual Range & 0.0052 & 0.0060 \\
Temperature Wettest Quarter & 0.0012 & 0.0012 \\
Temperature Driest Quarter & 0.0022 & 0.0015 \\
Temperature Warmest Quarter & 0.0008 & 0.0008 \\
Minimum Precipitation & 0.0028 & 0.0029 \\
Precipitation Warmest Quarter & 0.0037 & 0.0030 \\
Precipitation Coldest Quarter & 0.0034 & 0.0035 \\
\hline
\end{tabular}


bioRxiv preprint doi: https://doi.org/10.1101/2020.11.16.383877; this version posted November 17,2020 . The copyright holder for this preprint (which was not certified by peer review) is the author/funder, who has granted bioRxiv a license to display the preprint in perpetuity. It is made available under aCC-BY-NC-ND 4.0 International license.

Figure 1. Temperature annual range of North America and Central America from the WorldClim dataset (grayscale) with junco sampling locations for in situ measurements (red), phylogeography (blue), and acclimations (yellow).

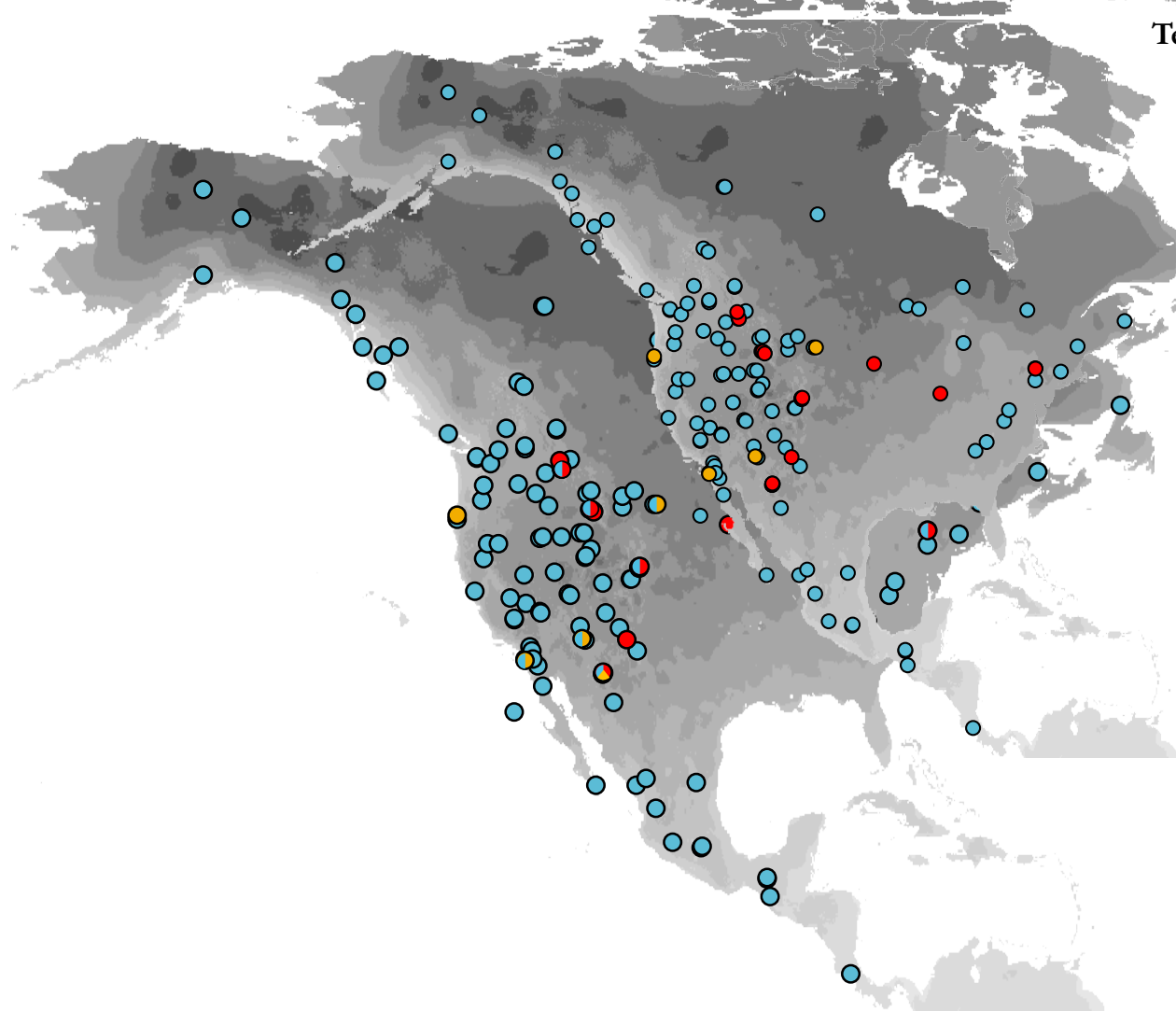

Temperature Annual Range $\left({ }^{\circ} \mathrm{C}\right)$

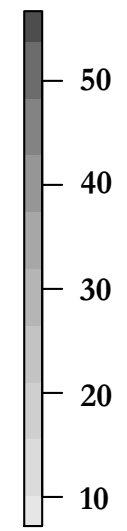


bioRxiv preprint doi: https://doi.org/10.1101/2020.11.16.383877; this version posted November 17,2020 . The copyright holder for this preprint (which was not certified by peer review) is the author/funder, who has granted bioRxiv a license to display the preprint in perpetuity. It is made available under aCC-BY-NC-ND 4.0 International license.

Figure 2. Effect of daily temperature range on in situ $\mathrm{M}_{\text {sum }}$ while controlling for differences in $\mathrm{M}_{\mathrm{b}}$. Model results shown in Table S3.

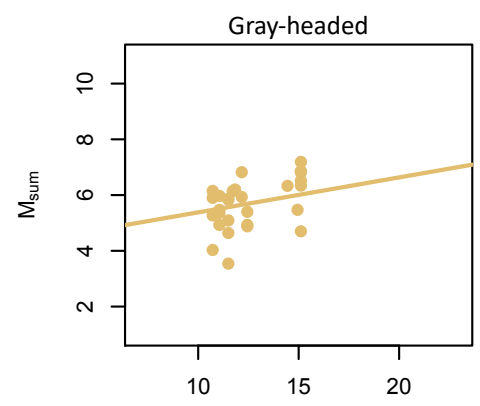

Daily Temperature Range

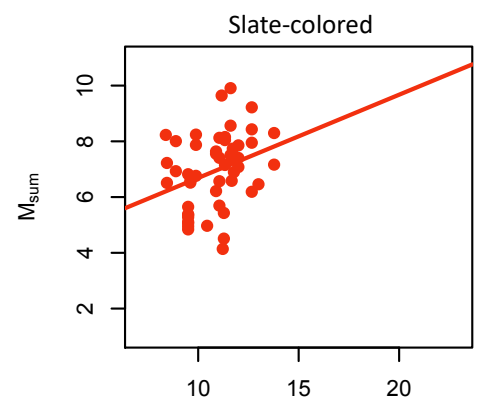

Daily Temperature Range



Daily Temperature Range



Daily Temperature Range



Daily Temperature Range 
Figure 3. Population genetic structuring along 4 PC axes (a,b), 4 RDA axes in unconditioned RDA (c,d), and 2 RDA axes in a conditioned model (e). Arrows indicate loadings of 8 WorldClim variables. Dots represent individuals, colors denote museum-based taxon assignments.

(a)



(c)

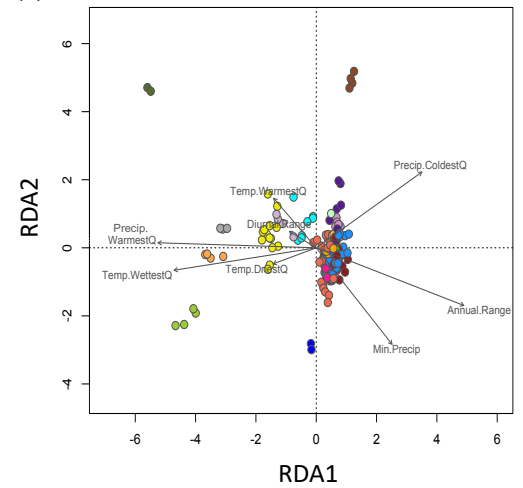

(b)



(d)

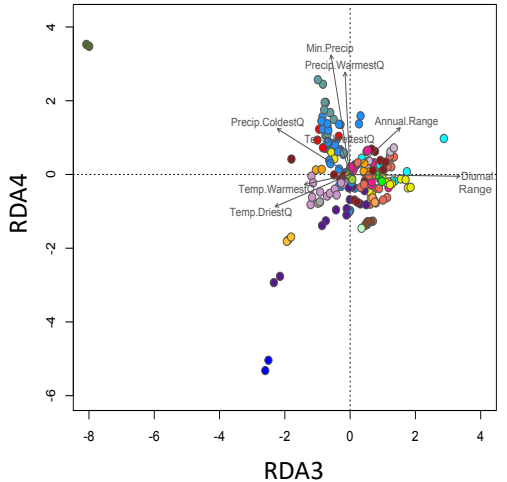

J. h. aikeni
J.h. caniceps

J. h. carolinensis

- J. h. cismontanus

- J.h. hyemalis

- J. h. mearnsi

J. h. oreganus

- J.h. oreganus

J. h. pontilis

- J. h. shufeldti

- J. h. thurberi

J. insularis

J. p. alticola

- J.p. fulves

- J. p. palliatus

J. p. phaeonotus
J. vulcani

(e)

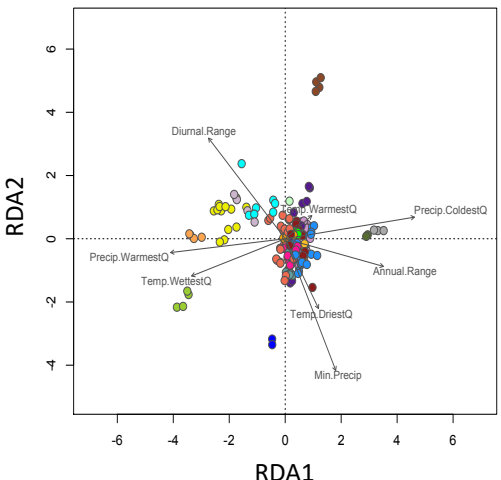


Figure 4. Change in $\mathrm{M}_{\text {sum }}$ (post- minus pre-acclimation) over a three-week acclimation period for each population ordered from lowest to highest native temperature range (from left to right). Control birds in red, cold-acclimated birds in blue; $n=95$.

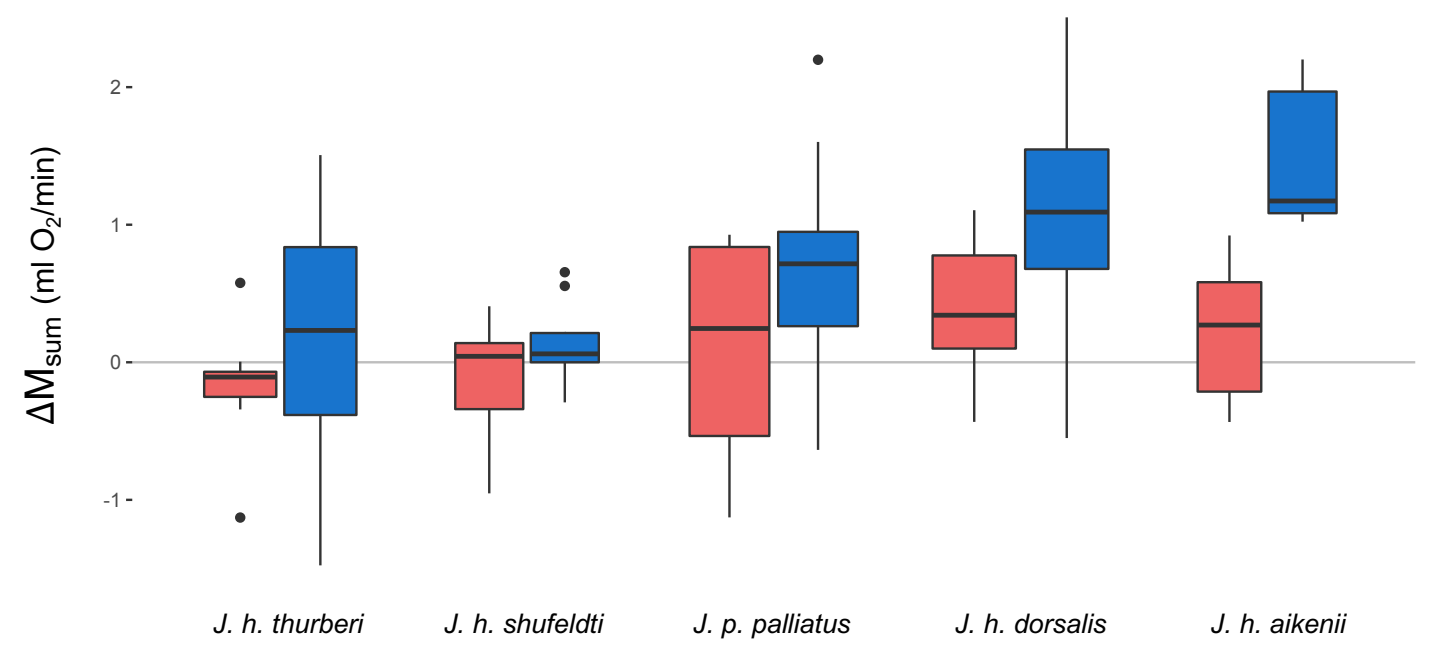

